\title{
DESIGUALDADES SOCIALES EN SALUD. \\ Un análisis de la mortalidad por las principales causas de muerte en España y Francia, 1988-1990
}

\author{
LOURDES LOSTAO
}

Universidad Pública de Navarra

\author{
ENRIQUE REGIDOR \\ Universidad Complutense de Madrid \\ PIERRE AÏACH \\ INSERM (Institut National de la Santé \\ et de la Recherche Médicale)
}

PALABRAS ClAVE ADICIONALES

ADDITIONAL KEYWORDS

Sociología de la Salud, Comparación Internacional, Diferencias Socioeconómicas.

Sociology of Health, International Comparison. Socioeconomic Differences.

RESUMEN. El objetivo de este artículo es comparar las desigualdades sociales en la mortalidad por las principales causas de muerte en España y en Francia durante el período 1988-90. Tanto en España como en Francia, la mortalidad en el grupo de trabajadores manuales es más alta que la mortalidad en el resto de los grupos ocupacionales para todas las causas de muerte analizadas. Por su parte, los agricultores presentan una mortalidad intermedia entre los profesionales y directivos y entre los trabajadores manuales. Los trabajadores manuales tienen una mortalidad 1,89 veces más alta que los profesionales y directivos en España y 1,67 veces más alta en Francia. En España, las mayores diferencias entre la mortalidad de trabajadores manuales y la mortalidad de profesionales y directivos se observa en accidentes y en las enfermedades del aparato respiratorio; por su parte, en Francia, las mayores diferencias se observan en las enfermedades del aparato digestivo y en las enfermedades del aparato respiratorio.

\section{INTRODUCCIÓN}

La investigación de las desigualdades en salud es un tema de gran interés ampliamente estudiado y tratado en Europa y en otros países. Puede decirse que la publicación del Informe Black en 1980 (Black,1980) promovió la investigación

Revista Internacional de Sociología (RIS)

Tercera Época, $\mathrm{n}^{\circ}$ 27, Septiembre-Diciembre, 2000, pp. 5-22. 
RIS

REVISTA INTERNACIONAL DE SOCIOLOGi.

No 27, Septiembre - Diciembre, 2000

LOURDES LOSTAO, ENRIQUE REGIDOR Y PIERRE AÏACH

acerca de las desigualdades en salud en Europa a lo largo de los ochenta y los noventa e inició un importante debate científico acerca de la naturaleza, dimensión y causas de las desigualdades en salud en las sociedades desarrolladas.

En este sentido, un conjunto amplio de investigaciones realizadas ha mostrado la existencia de una relación inversa entre nivel socioeconómico y mortalidad, de tal forma que los individuos pertenecientes a los estratos socioeconómicos más bajos presentan tasas de mortalidad superiores a las de los estratos socioeconómicos altos (Towsend y Davidson, 1982; Whitehead, 1988; Fox, 1989; Davey-Smith et al., 1990; Illsley y Svensson, 1990; Illsley y Wnuk-Lipinski, 1990; Pappas et al., 1993). La utilización de la mortalidad en la mayoría de estas investigaciones sobre desigualdades en salud se debe a que se trata del indicador más válido y fiable para reflejar el nivel de salud de la población. De hecho, las estadísticas de mortalidad continúan siendo ampliamente utilizadas en los países industrializados para la distribución de los recursos de salud, para la evaluación del impacto de determinados programas sanitarios y para la evaluación de las desigualdades en salud. En España, en cambio, la evidencia empírica acerca de las desigualdades sociales en salud con datos de mortalidad ha sido muy escasa (Regidor et al., 1995; 1996; Rodríguez y Lemkow, 1990). Sin embargo, y a pesar de las limitaciones, se han realizado investigaciones sobre desigualdades en salud (Durán, 1983; de Miguel et al., 1986; Rodríguez y Lemkow, 1990; Rodríguez y de Miguel, 1990), utilizando para ello información sobre distintas dimensiones de la misma, bien en todo el territorio español o, bien, en ámbitos geográficos más reducidos (Borrell y Arias, 1995; Arias y Borrell, 1998).

Según la Organización Mundial de la Salud, únicamente los indicadores de mortalidad presentan un grado de confianza adecuado para comparar el nivel de salud entre los distintos países. Además, la amplia disponibilidad de información sobre mortalidad en casi todos los países, junto al hecho de que se trata de un acontecimiento claramente definido, explican la gran utilización de las estadísticas sobre defunciones, en el ámbito sociosanitario. En este sentido, conviene señalar que un tipo de investigaciones que contribuye a esclarecer algunos de los factores macrosociales que producen las desigualdades sociales en mortalidad son las comparaciones internacionales. Estudios comparativos realizados con datos procedentes del inicio de la década de los ochenta en varios países europeos mostraron que la magnitud de las desigualdades sociales en mortalidad era diferente de unos lugares a otros dependiendo de la causa de muerte (Lundberg, 1986; Vägero y Lundberg, 1989; Valkonen, 1989; Leclerc et al., 1984; 1990; Kunst, 1997; Kunst et al., 1998; Mackenbach et al., 1997).

En cualquier caso, la mayor parte de estos estudios comparativos proceden, fundamentalmente, de Gran Bretaña y de los países nórdicos, debido a la ausencia de importantes series de datos en relación con este tema en la mayor parte de los países europeos (Illsley y Baker, 1991; Valkonen et al., 1991; 
Valkonen, 1993; Lahelma et al., 1994). Esta ausencia de datos se debe a que en el registro de mortalidad no suele recogerse la ocupación u otra variable que refleje el nivel socioeconómico de los fallecidos. A ello hay que unir la restricción impuesta por la legislación en la mayoría de los países a la utilización de los datos individualizados y personalizados, con lo que tampoco pueden estudiarse las características de los fallecidos enlazando el registro de defunciones con el censo de población, donde sí aparecen esas características socioeconómicas.

Por ejemplo, en España, debido al bajo nivel de cumplimentación de la ocupación en el Boletín Estadístico de Defunción (BED) y a las restricciones que impone la legislación, es imposible el estudio de las desigualdades sociales en mortalidad en el conjunto del Estado desde el inicio de la década de los ochenta. No obstante, una opción metodológica para valorar las diferencias socioeconómicas en mortalidad ha sido la utilización de los datos sobre fallecidos en las provincias donde el porcentaje de cumplimentación de la ocupación en el BED era alto. Esta ha sido, precisamente, la estrategia seguida por los autores del Estudio de las Ocho Provincias. Por este motivo, en la investigación que sirve de base a este artículo, se utilizan las defunciones según la ocupación procedentes del Estudio de las Ocho Provincias para comparar las desigualdades sociales en mortalidad en España y en Francia en el período 1988-90. En los estudios comparativos realizados con datos del inicio de los ochenta se observó que la mortalidad por enfermedades del corazón en los trabajadores manuales era similar a la de los trabajadores no manuales en ambos países, pero que la magnitud de las diferencias socioeconómicas en la mortalidad por otras causas de muerte era diferente de un país a otro (Mackenbach et al., 1997; Kunst et al., 1998).

\section{POBLACIÓN Y METODOLOGÍA}

El análisis se ha restringido a la población activa de varones de 25 a 64 años de edad en el período 1988-90. Los datos de defunciones se han obtenido de los registros de mortalidad de España y Francia. En el caso de España, los datos proceden del Estudio de las Ocho Provincias (Regidor et al., 1995), ya que esas son las provincias españolas en las que el porcentaje de ausencia de información sobre ocupación de los fallecidos en los certificados de defunción fue inferior al $10 \%$ en este período. Igualmente, el porcentaje de certificados de defunción en Francia sin la ocupación fue del $10 \%$ para este período. En el Estudio de las Ocho Provincias el número de fallecidos según la causa de muerte fué proporcionado por el Instituto Nacional de Estadística (INE), mientras que el número de fallecidos en Francia fue proporcionado por el Instituto Nacional Francés de la Salud (INSERM). La causa básica de la muerte es seleccionada 
y codificada usando la Clasificación Internacional de Enfermedades en su novena revisión (CIE-9).

En el Estudio de las Ocho Provincias la población a riesgo se obtuvo de la Encuesta de Población Activa realizada por el INE. Cada trimestre la muestra está integrada por 62.000 hogares, lo que supone unas 180.000 personas, con una tasa de no respuesta que oscila entre el 2 y el $3 \%$. En el presente estudio se han tomado las estimaciones de los varones de 25 a 64 años pertenecientes a la población económicamente activa de esas 8 provincias. Para usar esas estimaciones como denominadores se han convertido previamente en personas año. Dado que cada individuo en cada trimestre contribuye con un cuarto de año a la población a riesgo, el número de personas-años se ha obtenido dividiendo por 4 la suma de las 12 estimaciones trimestrales de cada período analizado. Por su parte, la población a riesgo para Francia se ha tomado del Censo de Población de 1990 y ha sido proporcionada por el Instituto Nacional de la Estadística y Estudios Económicos (INSEE). Para estimar el número de personas-años a riesgo en cada período se ha multiplicado por 3 las estimaciones de varones de 25 a 64 años económicamente activos.

Los individuos fueron agrupados en cuatro grupos o categorías ocupacionales. La clasificación de la ocupación que figura en el registro de mortalidad de España determinó los grupos ocupacionales utilizados, ya que en ese registro sólo se emplea el primer dígito de la Clasificación Internacional de Ocupaciones (CIO) de 1968, con lo que la ocupación es asignada a alguno de los siguientes grandes grupos: profesionales, técnicos y similares; directivos y gerentes; personal de los servicios administrativos; comerciantes y vendedores; personal de los servicios de hostelería, domésticos y de seguridad; agricultores y similares; trabajadores de la industria, la construcción y los transportes.

Para el presente análisis comparativo, los individuos procedentes del Estudio de las Ocho Provincias se han clasificado en los siguientes 4 grupos: el grupo de profesionales y directivos, compuesto por la suma del primer y segundo grupo de la CIO; el grupo de los trabajadores de los servicios compuesto por la suma del tercer, cuarto y quinto grupos de la CIO; el grupo de agricultores que corresponde al sexto grupo de la $\mathrm{CIO}$, y el grupo de trabajadores manuales el cual corresponde al séptimo grupo de la CIO.

Por su parte, en el registro de mortalidad de Francia la ocupación de los fallecidos durante el período de vida económicamente activa aparece codificada en alguna de las 32 categorías de la Clasificación de Categorías Socio-Profesionales. Los fallecidos incluidos en cada una de estas categorías fueron asignados a alguno de los 7 grandes grupos de la CIO y, posteriormente, fueron agrupados en los cuatro grupos señalados anteriormente.

Se han valorado las diferencias socioeconómicas en mortalidad por distintas enfermedades en el grupo de 25 a 64 años en España y en Francia. La evaluación de las diferencias en mortalidad entre estos cuatro grupos se ha realizado 
mediante la estimación del riesgo relativo de mortalidad estandarizado por edad. El cálculo de los riesgos relativos de mortalidad se ha realizado con la utilización de modelos de regresión log-lineales de Poisson, tomando como referencia el grupo de profesionales y directivos.

Posteriormente se han evaluado las diferencias en mortalidad entre los trabajadores manuales y los trabajadores no manuales, excluyendo del análisis a los agricultores. Para ello se han incluido en un solo grupo de trabajadores no manuales a los profesionales y directivos y a los trabajadores de los servicios. Las diferencias en mortalidad en estos dos grupos se han evaluado como en el caso anterior mediante el riesgo relativo de mortalidad ajustado por edad.

\section{RESULTADOS}

El número total de defunciones en cada uno de los cuatro grupos ocupacionales por las diferentes causas de muerte estudiadas en España y Francia, así como el número de personas-año a riesgo, aparecen en la tabla 1 .

En la tabla 2 se exponen los riesgos relativos de mortalidad ajustados por edad por las distintas causas de muerte analizadas en España en el período 1988-90. La mortalidad en el grupo de trabajadores manuales fue siempre más alta que la mortalidad en el resto de los grupos ocupacionales. El riesgo relativo de la mortalidad por el conjunto de las causas de muerte en el grupo de trabajadores manuales con respecto a la del grupo de profesionales y directivos fue de 1,89. El tamaño del riesgo relativo de mortalidad fué diferente, dependiendo de las causas de muerte: 3,35 en accidentes, 3,33 en enfermedades del aparato respiratorio, 2,14 en enfermedades del aparato digestivo, 1,56 en enfermedades cardiovasculares y 1,51 en cáncer.

La mortalidad en los agricultores fué de una magnitud intermedia entre la del grupo de trabajadores no manuales-profesionales y directivos y trabajadores de los servicios y la del grupo de trabajadores manuales. El riesgo relativo de mortalidad para el conjunto de todas las causas de muerte fue 1,70. Por su parte, los trabajadores de los servicios tuvieron también un riesgo relativo de mortalidad mayor que los profesionales y directivos para el conjunto de todas las causas de muerte $(1,10)$. En cambio, la mortalidad por cáncer, la mortalidad por enfermedades cardiovasculares y la mortalidad por accidentes no presentó diferencias estadísticamente significativas con respecto a la mortalidad de los profesionales y directivos.

En la tabla 3 se muestran los riesgos relativos de mortalidad ajustados por edad para las distintas causas de muerte analizadas en Francia en el período 1988-90. Al igual que ocurre en España, la mortalidad en el grupo de trabajadores manuales fue siempre más alta que en el grupo de profesionales y directivos y que el resto de los grupos ocupacionales para todas las causas de muerte 
Tabla 1.

Número total de defunciones según grupo ocupacional y causa de muerte y número de personas-año a riesgo en España* y Francia. Población activa de varones de 25 a 64 años. Periodo 1988-1990.

\begin{tabular}{|c|c|c|c|c|c|c|}
\hline Causa de muerte $\operatorname{CIE}$ ( $9^{\mathrm{a}}$ edición) & $\begin{array}{c}\text { Todas } \\
\text { las causas } \\
001-999\end{array}$ & $\begin{array}{c}\text { Cáncer } \\
\text { 140-208 }\end{array}$ & $\begin{array}{c}\text { Enfermedades } \\
\text { Aparato } \\
\text { Circulatorio } \\
390-459\end{array}$ & $\begin{array}{c}\text { Enfermedades } \\
\text { Aparato } \\
\text { Digestivo } \\
520-579\end{array}$ & $\begin{array}{c}\text { Enfermedades } \\
\text { Aparato } \\
\text { Respiratorio } \\
460-519\end{array}$ & $\begin{array}{c}\text { Accidentes } \\
800-899\end{array}$ \\
\hline \multicolumn{7}{|l|}{ ESPAÑA* } \\
\hline Profesionales y directivos & 562 & 232 & 161 & 46 & 14 & 59 \\
\hline Trabajadores de los servicios & 1.644 & 606 & 420 & 178 & 75 & 267 \\
\hline Agricultores & 2.930 & 1.043 & 935 & 241 & 150 & 375 \\
\hline Trabajadores manuales & 5.192 & 1.701 & 1.222 & 474 & 229 & 982 \\
\hline \multicolumn{7}{|l|}{ Número de personas-año 2.798} \\
\hline \multicolumn{7}{|l|}{ FRANCIA } \\
\hline Profesionales y directivos & 18.752 & 6.918 & 3.521 & 739 & 324 & 1.699 \\
\hline Trabajadores de los servicios & 46.711 & 17.189 & 8.736 & 2.815 & 987 & 4.058 \\
\hline Agricultores & 13.719 & 4.773 & 2.978 & 896 & 406 & 870 \\
\hline Trabajadores manuales & 68.162 & 27.149 & 11.344 & 4.996 & 1.782 & 6.023 \\
\hline Número de personas-año 12.323 & & & & & & \\
\hline
\end{tabular}

* Estudio de las Ocho Provincias. 


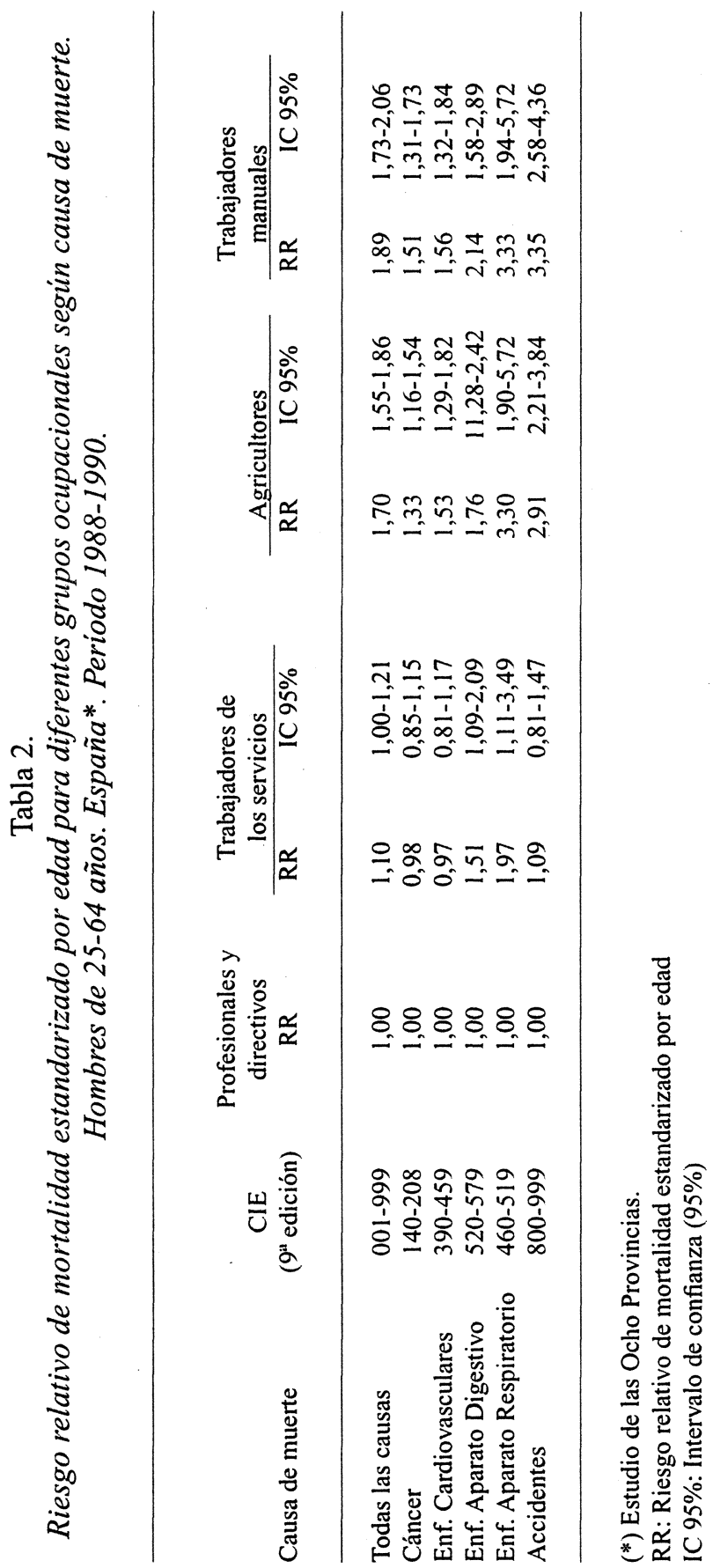


Riesgo relativo de mortalidad estandarizado por edad para diferentes grupos ocupacionales según causa de muerte. Hombres de 25-64 años. Francia. Período 1988-1990.

\begin{tabular}{|c|c|c|c|c|c|c|c|c|}
\hline \multirow[b]{2}{*}{ Causa de muerte } & \multirow{2}{*}{$\begin{array}{c}\text { CIE } \\
\text { (9a edición) }\end{array}$} & \multirow{2}{*}{$\begin{array}{c}\begin{array}{c}\text { Profesionales y } \\
\text { directivos }\end{array} \\
\text { RR }\end{array}$} & \multicolumn{2}{|c|}{$\begin{array}{c}\text { Trabajadores } \\
\text { servicios }\end{array}$} & \multicolumn{2}{|c|}{ Agricultores } & \multicolumn{2}{|c|}{$\begin{array}{c}\text { Trabajadores } \\
\text { manuales }\end{array}$} \\
\hline & & & $\mathrm{RR}$ & IC $95 \%$ & RR & IC $95 \%$ & $\mathrm{RR}$ & IC $95 \%$ \\
\hline Todas las causas & 001-999 & 1,00 & 1,42 & $1,40-1,45$ & 1,45 & $1,41-1,48$ & 1,67 & $1,65-1,70$ \\
\hline Cáncer & $140-208$ & 1,00 & 1,39 & $1,36-1,43$ & 1,20 & $1,15-1,24$ & 1,85 & $1,80-1,90$ \\
\hline Enfermedades cardiovasculares & $390-459$ & 1,00 & 1,40 & $1,34-1,45$ & 1,51 & $1,43-1,58$ & 1,52 & $1,45-1,57$ \\
\hline Enfermedades aparato digestivo & $520-579$ & 1,00 & 2,16 & $1,99-2,34$ & 2,24 & $2,03-2,48$ & 3,19 & $2,95-3,45$ \\
\hline Enfermedades aparato respiratorio & o $460-519$ & 1,00 & 1,70 & $1,50-1,93$ & 2,12 & $1,83-2,46$ & 2,57 & $2,28-2,89$ \\
\hline Accidentes & $800-999$ & 1,00 & 1,42 & $1,34-1,50$ & 1,43 & $1,32-1,55$ & 1,53 & $1,45-1,61$ \\
\hline
\end{tabular}

$\left({ }^{*}\right)$ Estudio de las Ocho Provincias.

RR: Riesgo relativo de mortalidad estandarizado por edad.

IC 95\%: Intervalo de confianza (95\%). 
analizadas. El riesgo relativo de mortalidad para el conjunto de las causas de muerte en el grupo de trabajadores manuales con respecto a la del grupo de profesionales y directivos fue de 1,67. Los riesgos relativos de mortalidad en las causas de muerte analizadas fueron: 1,53 en accidentes; 2,57 en enfermedades del aparato respiratorio; 3,19 en enfermedades del aparato digestivo; 1,52 en enfermedades cardiovasculares y 1,85 en cáncer.

Los agricultores tuvieron un riesgo relativo de mortalidad para el conjunto de todas las causas, de 1,45. Como en España, la mortalidad de los agricultores franceses por las distintas causas de muerte presentó una magnitud intermedia entre la mortalidad de los trabajadores no manuales y la de los trabajadores manuales. Por otra parte, los trabajadores de los servicios tuvieron también un riesgo relativo de mortalidad mayor que los profesionales y directivos para el conjunto de todas las causas de muerte $(1,42)$; además, en todas las causas de muerte presentaron una mortalidad mayor que la mortalidad de los profesionales y directivos.

Hay que señalar que en España, la magnitud del riesgo relativo en los agricultores y en los trabajadores manuales fue superior a la de Francia - excepto en la mortalidad de las enfermedades del aparato digestivo en ambos grupos y en la mortalidad por cáncer en los trabajadores manuales-; en cambio, la magnitud del riesgo relativo en los trabajadores de los servicios fue siempre superior en Francia que en España.

Finalmente, en la Figura 1 aparecen los riesgos relativos de mortalidad entre el grupo de trabajadores manuales y el grupo de trabajadores no manuales para las causas de muerte analizadas. La magnitud de ese riesgo relativo fue mayor en España que en Francia, excepto en las enfermedades del aparato digestivo.

\section{DISCUSIÓN}

La investigación realizada muestra unos resultados similares a los obtenidos en otros estudios efectuados en diferentes países: una relación inversa entre mortalidad y nivel socioeconómico, esto es, menor mortalidad en los estratos socioeconómicos altos. Algunos autores han señalado que estas diferencias se deben a una mayor exposición a factores de riesgo para la salud en los estratos socioeconómicos bajos, como el consumo excesivo de alcohol, el tabaquismo, la hipertensión, la inactividad fisica, la exposición a agentes físicos y químicos para la salud en el medio ambiente laboral, el grado de control y autonomía en el puesto de trabajo, etc. En cambio, para otros autores lo más importante para la salud es la posición que se ocupe en la jerarquía socioeconómica. Según esta hipótesis las personas de los grupos socioeconómicos más altos tienen mayor capacidad para amortiguar los acontecimientos estresantes para su salud y para 
R I S

REVISTA INTERNACIONAL UE SOCIOLOCIA

Figura 1.

Riesgo relativo de mortalidad entre trabajadores manuales y no manuales. Población activa de varones de 25 a 64 años. España y Francia, 1988-90.

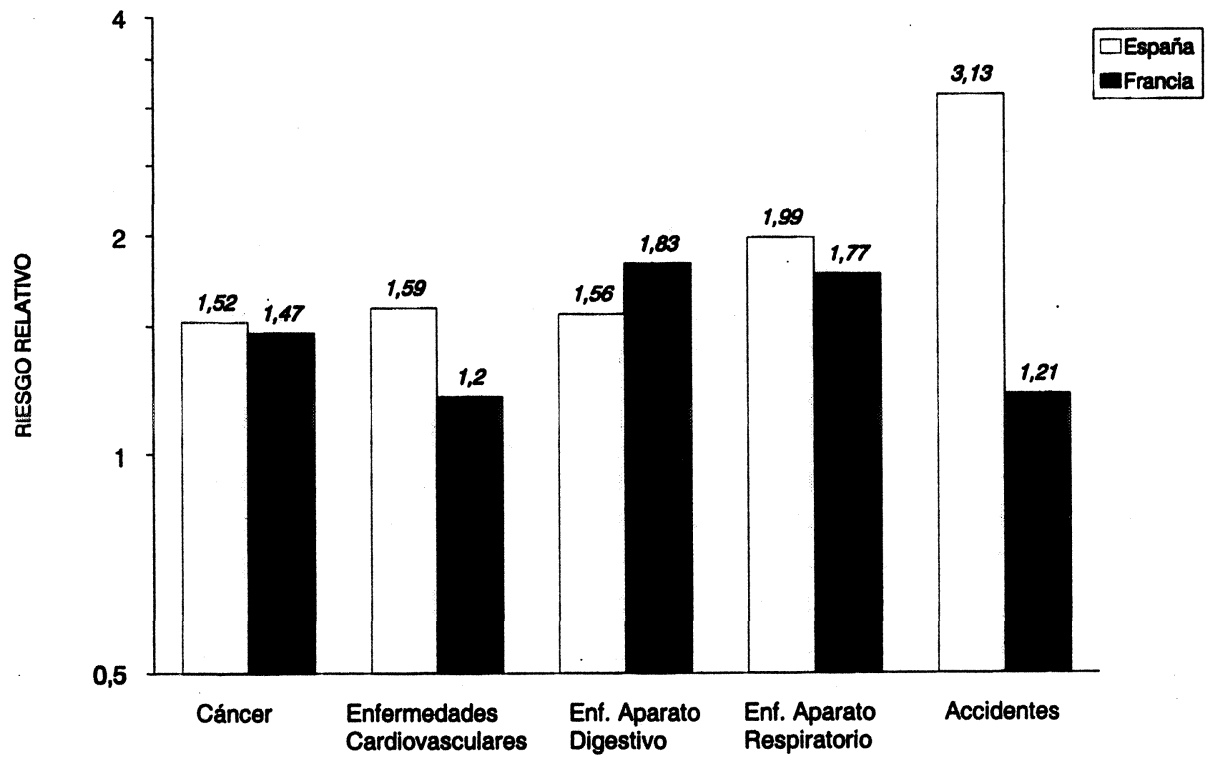

el afrontamiento de los mismos, lo que les permite un mejor conocimiento de los factores protectores y de riesgo de enfermedades, así como la capacidad de actuar en consecuencia con estilos de vida positivamente asociados con la salud, como son el bajo consumo de tabaco y alcohol, realizar ejercicio físico, llevar una dieta alimenticia adecuada, asistencia a screenings,etc... (Adler et al., 1994; Evans et al., 1994).

Otra de las explicaciones expuestas a la hora de interpretar las. diferencias socioeconómicas en mortalidad encontradas en los estudios transversales es la que se refiere a la posible existencia de un sesgo de selección, según el cual es el estado de salud de los individuos el que determina su posición social en lugar de ser al contrario (Blane, 1985; 1993; Regidor et al., 1994). De esta forma, puede ocurrir que los individuos menos saludables se concentren en los estratos socioeconómicos más bajos al final de su vida económicamente activa o que el estado de salud durante la infancia determine las desigualdades sociales en salud en la época adulta. Sin embargo, diferentes estudios longitudinales han concluido que la movilidad social durante la vida activa de los individuos contribuye muy poco a las desigualdades sociales en mortalidad (Fox et al., 1986; 
Goldblatt, 1989). Así mismo, como se ha reflejado en la literatura científica, ciertos factores tales como el bienestar material en la infancia, el nivel de estudios alcanzado y/o las características de personalidad de los individuos (West, 1991; Davey Smith et al., 1994; Ross y Wu, 1995; Reynolds y Ross, 1998) pueden influir tanto en el nivel socioeconómico futuro como en el nivel de salud. Por último, una teoría reciente señala al nivel socioeconómico como la causa fundamental de las diferencias socioeconómicas en salud, ya que según los autores son las condiciones sociales las que exponen a los individuos a los riesgos para la salud (Link y Phelan, 1995). Según dichos autores, factores sociales como la situación socioeconómica y el apoyo social son probables "causas fundamentales" de enfermedad, que debido a que representan acceso a recursos importantes, afectan a las consecuencias de múltiples enfermedades a través de diferentes mecanismos, y consecuentemente mantienen una asociación con la enfermedad incluso cuando cambian los mecanismos intervinientes. La característica esencial de las causas sociales fundamentales, es que conllevan acceso a recursos que pueden ser utilizados para evitar riesgos o para minimizar las consecuencias de la enfermedad una vez que se presenta. Sin una cuidadosa atención a estas posibilidades, correríamos el riesgo de imponer estrategias de intervención basadas en el individuo que son ineficaces, y se perderían oportunidades para adoptar intervenciones sociales de base más amplia que podrían producir importantes beneficios sanitarios para los ciudadanos.

\section{CONCLUSIONES}

En el presente estudio se ha observado que la magnitud de las desigualdades sociales en mortalidad en España y en Francia varía dependiendo de la causa de muerte. En el caso de las enfermedades del aparato circulatorio, el riesgo relativo de mortalidad en agricultores y trabajadores manuales es similar en ambos países. Las desigualdades sociales en la mortalidad por enfermedad cardiovascular es un fenómeno generalizado en las sociedades industrializadas y distintos estudios señalan que los hombres pertenecientes a grupos de niveles socioeconómicos más bajos tienen más probabilidad de morir de enfermedad cardiovascular. Estas diferencias se han atribuido a que los grupos socioeconómicos más bajos están más frecuentemente expuestos a otros factores de riesgo de estas enfermedades, como consumo excesivo de alcohol, hipertensión, consumo de tabaco, sobrepeso (Kaplan y Keil, 1993) y a mayor exposición a estrés laboral en los puestos de trabajo (Karasek et al., 1988; Freund y McGuire, 1995). Otra posible hipótesis explicativa que se ha dado es que los grupos socioeconómicos más bajos tienen menos acceso a una utilización menos efectiva de los servicios que son importantes para la detección precoz, como es el control de la hipertensión (Casper et al., 1992; O'Brian et al., 1982; Schnall 
R I S

et al., 1992). En España, curiosamente, los trabajadores de los servicios tenían menor riesgo de mortalidad que los profesionales y directivos. Como una posible explicación de este hallazgo hay que señalar una menor prevalencia de la hipertensión en este grupo ocupacional. De hecho, en 1987 los hombres pertenecientes a las clases sociales intermedias tenían menor frecuencia de hipertensión que los profesionales y directivos y los trabajadores manuales (Comisión Científica de las Desigualdades Sociales 1996, Ministerio de Sanidad y Consumo). Igualmente tampoco hay que descartar la influencia que ha podido tener el consumo de alcohol. Así, según la Encuesta Nacional de Salud de 1987, el grupo de los trabajadores de los servicios era el grupo ocupacional con un menor consumo excesivo de alcohol (Encuesta Nacional de Salud, Ministerio de Sanidad y Consumo, 1987). Otras explicaciones que se han dado acerca de las desigualdades sociales en mortalidad por enfermedades cardiovasculares podrían deberse a circunstancias relacionadas con la ausencia de información respecto a la salud y de los valores o normas relativas a las conductas relacionadas con la salud. El desigual acceso de todos los estratos de la población a la información sanitaria acerca de los factores de riesgo de la enfermedad cardiovascular, así como del resto de las causas de muerte analizadas, influye en que los grupos socioeconómicos más altos sean probablemente los mejor informados y tiendan a modificar sus estilos de vida, así como sus condiciones de vida con el objeto de minimizar los riesgos para su salud.

Diferentes estudios comparativos previos (Kunst, 1997; Kunst et al., 1998 y 1999) han señalado que en España y Francia los trabajadores no manuales tenían en los años ochenta una mortalidad por enfermedad cardiovascular superior a la de los trabajadores manuales. En los años noventa, el presente estudio muestra una inversión de esta relación en ambos países. Otros estudios (Regidor et al., 1995; Lang y Ducimetière, 1995) ya habían señalado una inversión de esta relación a lo largo de los ochenta en España y en Francia. Este cambio en el patrón de la mortalidad por esta enfermedad en hombres, en relación con el nivel socioeconómico, se produjo alrededor de los años sesenta en países como Reino Unido (Marmot et al., 1978) y Holanda (Mackenbach, 1992) o en los setenta, como Suecia (Vagerö, 1991), así como en otros países (Valkonen, 1982; Kristofersen, 1979; Lynge, 1979; Doornbos, 1990). La demora de la inversión en España y en Francia pudiera atribuirse a un retraso en la relación entre factores de riesgo de esta enfermedad y clase social al igual que ocurrió en otros países como EEUU décadas antes. Así por ejemplo, en estos países a final de los cincuenta y los sesenta las clases sociales altas disminuyen el consumo de tabaquismo y aumenta su consumo en las clases bajas (Vagerö et al., 1989; Van Reek et al., 1988). Esta situación se ha producido más tarde en los países del sur de Europa (Sasco et al., 1994; Comisión Científica de las Desigualdades Sociales, 1996) y posiblemente explique la demora en la inversión de la relación observada en estos países. 
Más difícil es argumentar alguna explicación para los resultados encontrados en la mortalidad por cáncer, dado que se trata de un grupo heterogéneo de enfermedades con multitud de factores causales y, en muchos casos, poco conocidos. No obstante, la mayor frecuencia de tabaquismo en las clases sociales bajas también puede estar implicada en estos resultados, debido a que el cáncer de pulmón, fuertemente asociado al consumo de tabaco, es el cáncer más común en los hombres. En cuanto a las enfermedades del aparato digestivo, la magnitud de las desigualdades sociales en mortalidad es mayor en Francia que en España. Igualmente, en otros estudios que han comparado las desigualdades sociales en mortalidad por enfermedades del aparato digestivo en varios países, también encontraron mayor desigualdad en Francia (Kunst et al.,1998). La mayoría de los autores han atribuido estos resultados a la elevada mortalidad por cirrosis hepática en los estratos socioeconómicos bajos como consecuencia del excesivo consumo de alcohol en las clases sociales bajas en Francia.

Por lo que se refiere a la mortalidad por enfermedades del aparato respiratorio y por accidentes, las desigualdades sociales son mayores en España que en Francia. En la mortalidad por enfermedades del aparato respiratorio, las mayores desigualdades sociales en España posiblemente se deban a que durante los años ochenta se observó en España una mayor diferencia en el consumo de tabaco entre las clases sociales que en Francia. $Y$ en cuanto a la mortalidad por accidentes, hay que señalar a los accidentes de tráfico y a los accidentes relacionados con el trabajo y a la importancia que tienen las condiciones materiales y medioambientales en su ocurrencia. En este sentido, es posible que las clases socioeconómicas bajas en España durante el período de estudio estuvieran expuestas a peores condiciones materiales y/o medioambientales que en Francia: vehículos peor equipados en cuanto a seguridad, carreteras y vías urbanas en peores condiciones, mayor exposición a noxas fisicas y químicas en los lugares de trabajo, etc.

Finalmente, en la interpretación de los resultados obtenidos en este estudio hay que tener en cuenta algunas limitaciones. En primer lugar, esta investigación está restringida a hombres, con lo que los resultados no pueden ser aplicados a mujeres. Así mismo, sólo se ha estudiado la población activa de varones y se ha excluido a la población económicamente inactiva, con lo que las diferencias socioeconómicas en mortalidad estarán infraestimadas, ya que las personas excluídas pertenecen a los grupos con las tasas de mortalidad más altas (Vagerö, 1991; Dahl, 1993). Otra posible limitación es la existencia de un sesgo numerador/denominador, ya que las defunciones y las poblaciones a riesgo proceden de distintas fuentes de información (OPCS, 1978). No obstante, cuando ese sesgo se ha eliminado mediante la realización de algún estudio de carácter longitudinal (Fox et al., 1986), se ha comprobado que su efecto es más teórico que real, ya que no se han encontrado grandes variaciones en las diferencias socioeconómicas en mortalidad frente a las obtenidas con los 
RIS

REVISTA INTERNACIONAL DE SOCIOLOCIA

N*27, Septiembre - Diciembre, 2000

LOURDES LOSTAO, ENRIQUE REGIDOR Y PIERRE AÏACH

estudios transversales. Por último, cabe la posibilidad de que en la mortalidad por ocupación las ocho provincias estudiadas no representen la mortalidad por ocupación en el conjunto de la población. Sin embargo, los resultados obtenidos en estas provincias en los años ochenta (Regidor et al., 1995 y 1996) son similares a los que se obtuvieron cuando se analizaron las defunciones del conjunto de la población española (Regidor et al., 1994).

En resumen, en la presente investigación se ha puesto de manifiesto la existencia de desigualdades sociales en mortalidad por diferentes causas de muerte en España y en Francia. Así mismo se ha constatado que tanto en España como en Francia, la mortalidad en el grupo de trabajadores manuales fue siempre más alta que la mortalidad en el resto de los grupos ocupacionales para todas las causas de muerte analizadas. Por otro lado, esta investigación presenta unos resultados similares a los obtenidos en otros estudios realizados en distintos países, mostrando la existencia de una relación inversa entre mortalidad y nivel socioeconómico, esto es, menor mortalidad en los estratos socioeconómicos altos. Posteriormente, hemos observado también que la magnitud de las desigualdades sociales en mortalidad en España y en Francia varía según la causa de muerte. Además, se pone de manifiesto la importancia que tiene la utilización de varios grupos ocupacionales en este tipo de investigaciones en lugar de los dos grandes grupos de trabajadores manuales y no manuales. Siguiendo esta línea, se ha comprobado la existencia y magnitud de desigualdades sociales en el nivel de salud de la población de ambos países. La magnitud y las características de las desigualdades sociales en mortalidad señalan que éstas deben ser consideradas como un problema de salud pública de primer orden, así como que su reducción o eliminación debería figurar en cualquier agenda de política sanitaria que tenga como fin último mejorar el nivel de salud de la comunidad.

\section{BIBLIOGRAFÍA}

ADLER N.E., T. BOYCE, M.A. CHESNEY, S. COHEN, S. FOLKMAN, R.L. KAHN y S.L. SYME (1994), "Socioeconomic status and health: the challenge of the gradient", American Psychologist, $\mathrm{n}^{\circ} 49$, pp. 15-24.

ARIAS L.C. y C. BORRELL (1998), "Desigualdades en la mortalidad según la educación en la ciudad de Barcelona", Medicina Clínica (Barc), n¹10, pp. 161-166.

BLACK, D. (1980), Inequalities in Health, Department of Health and Social Security, London.

BLANE, D. (1985), "An assessment of the Black Report's explanations of health inequalities", Sociology of Health and Illness, $\mathrm{n}^{\circ} 7$, pp. 423-445.

BLANE, D., G. DAVEY SMITH y M. BARTLEY (1993), "Social selection: what does it contribute to social class differences in health?", Sociology of Health and Illness, $n^{\circ} 15, \mathrm{pp} .1-15$. 
BORRELL C. y A. ARIAS (1995), "Socio-economic factors and mortality in urban settings: the case of Barcelona (Spain)", Journal of Epidemiology and Community Health, $\mathrm{n}^{\circ} 49$, pp. $460-465$.

CASPER M., S. WING, D. STROGATZ, C.E. DAVIS y H.A. TYROLER (1992), "Antihypertensive treatment and US trends in stroke mortality, 1962 to 1980", American Journal of Public Health, $\mathrm{n}^{\circ} 82$, pp. 1600-1606.

COMISIÓN CIENTÍFICA DE ESTUDIOS DE LAS DESIGUALDADES SOCIALES EN SALUD EN ESPAÑA (1996), Desigualdades sociales en salud en España, Ministerio de Sanidad y Consumo, Madrid.

DAHL, E. (1993), "Social inequality in health. The role of the healthy worker effect", Social Science and Medicine, $\mathrm{n}^{\circ} 36, \mathrm{pp}$. 1077-1086.

DAVEY SMITH, G., M. BARTLEY y D. BLANE (1990), "The Black Report on socioeconomic inequalities in health 10 years on", British Medical Journal, n" 301, pp. 373-377.

DAVEY SMITH,G., D. BLANE y M. BARTLEY (1994), "Explanations for socio-economic differentials in mortality", European Journal of Public Health, n 4, pp. 131-144.

DOORNBOS G. y D. KROMHOUT (1990), "Educational level and mortality in a 32-year follow-up study of 18-year old men in the Netherlands", International Journal of Epidemiology, $n^{\circ} 19$, pp. 374-379.

DURÁN, M.A. (1983), Desigualdad social y enfermedad, Madrid, Tecnos.

DE MIGUEL J.M., L. LEMKOW y J.A. RODRÍGUEZ (1986), "Social inequities related to health in Spain", The Health Burden of Social Inequities, pp. 171-185, Copenhagen.

ENCUESTA NACIONAL DE SALUD (1987), Ministerio de Sanidad y Consumo, Madrid.

EVANS, R.G., M.L. BARER y T.R. MARMOR (1994), Why are some people healthy and others not?: The determinants of health of populations, New York, De Gruyter.

FOX, J. (Ed.) (1989), Health Inequalities in European Countries, Gower, Aldershot.

FOX, A.J., P. GOLDBLATT y D.R. JONES (1986), "Social class mortality differentials: artefact, selection or life circumstances?", en R.G. Wilkinson (Ed.), Class and Health. Research and longitudinal data, pp. 34-49, Londres, Tavistock Publications.

FREUND, P.E.S. y M.B. MCGUIRE (1995), Health, Illness and the Social Body, Englewood Cliffs, NJ, Prentice-Hall.

GOLDBLATT, P. (1989), "Mortality by social class, 1971-85", Population Trends, $\mathrm{n}$ " 56, pp. 6-15.

ILLSLEY R. y D. BAKER (1991), "Contextual variations in the meaning of health inequality", Social Science and Medicine, n 32, pp. 359-365. 


\section{RIS}

ILLSLEY, R. y P.G. SVENSSON (1990), Número monográfico sobre "Health equities in Europe", Social Science and Medicine, $\mathrm{n}^{\circ} 27, \mathrm{pp} .223-430$.

ILLSLEY, R. y Z. WNUK-LIPINSKI (1990), Número monográfico sobre "Non-market economic in health", Social Science and Medicine, $\mathrm{n}^{\circ} 27, \mathrm{pp} .831-889$.

KAPLAN, G.A. y J.E. KEIL (1993), "Socioeconomic factors and cardiovascular disease: a review of the literature", Circulation, nº 88, pp. 1973-1997.

KARASEK, R., T. THEORELL, J. SCHWARTZ, P. SCHNALL, C. PIEPER y J. MICHELA (1988), "Job Characteristics in Relation to the Prevalence of Myocardial Infarction in the US Health Examination Survey (HES) and the Health and Nutrition Examination Survey (HANES), American Journal of Public Health, nº 78, pp. 910-918.

KRISTOFERSEN, L. (1979), Occupational Mortality, Oslo, Norway, Central Bureau of Statistics.

KUNST, A.E. (1997), Cross-national comparisons of socio-economic differences in mortality, Tesis Erasmus, University Rotterdam.

KUNST, A.E., F. GROENHOF y J.P. MACKENBACH (1998), "Occupational class and cause specific mortality in middle aged men in 11 European countries: comparison of population based studies", British Medical Journal, nº 316, pp. 1636-1642.

KUNST, A.E. et al (1999), "Occupational Class and Ischemic Heart Disease Mortality in the United States and 11 European Countries", American Journal of Public Health, n89, pp. 47-53.

LAHELMA E., K. MANDERBACKA, O. RAHKONEN y A. KARISTO (1994), "Comparisons of inequalities in health: evidence from national surveys in Finland, Norway and Sweden", Social Science and Medicine, $\mathrm{n}^{\circ}$ 38, pp. 517-524.

LANG, T. y P. DUCIMETIÈRE (1995), "Premature Cardiovascular Mortality in France: Divergent Evolution between Social Categories from 1970 to 1990", International Journal of Epidemiology, n"24, pp. 331-339.

LYNGE, E. (1979), "Occupational mortality in Denmark 1970-1975", Scand Pop Stud, n" 5, pp. 120-130.

LINK, B.G. y J. PHELAN (1995), "Social conditions as fundamental causes of disease", Journal of Health and Social Behavior, número extra, pp. 80-94.

LECLERC, A., F. LERT y C. FABIEN (1990), "Differential Mortality: Some Comparisons between England and Wales, Finland and France, Based on Inequality Measures", International Journal of Epidemiology, $\mathrm{n}^{\mathrm{0}}$ 19, pp. 1001-1010

LECLERC, A., F. LERT y M. GOLDBERG (1984), "Les inegalites sociales devant la mort en Grande Bretagne et en France", Social Science and Medicine, $\mathrm{n}^{\circ}$ 19, pp. 479-487.

LUNDBERG, O. (1986), "Class and Health: Comparing Britain and Sweden”, Social Science and Medicine, $\mathrm{n}^{\circ} 23$, pp. 511-517. 
MACKENBACH, J.P. (1992), "Socio-economic health differences in the Netherlands: a review of recent empirical findings", Social Science and Medicine, $\boldsymbol{n}^{\mathrm{t}} 34$, pp. 213-226.

MACKENBACH, J.P., A.E. KUNST, A.E.J.M. CAVELAARS, F. GROENHOF y J.J.M. GEURTS, (1997), EU Working Group on Socio-economic Inequalities in Healt, "Socio-economic inequalities in morbidity and mortality in Western Europe: a comparative study", Lancet, $\mathrm{n}^{\circ} 349$, pp. 1655-1659.

MARMOT, M.G., A. M. ADELSTEIN, N. ROBINSON y G.A. ROSE (1978), "Changing social class distribution of heart disease", British Medical Journal, $\mathrm{n}^{\circ} 2,1109-1112$.

O'BRIAN SMITH E., J.D. CURB, R.J. HARDY, M.C. HAWKINS y H.A. TYROLER (1982), "Clinic attendance in the hypertension detection and follow-up program", Hypertension, $n^{\circ} 4,710-715$.

OFFICE OF POPULATION CENSUSES AND SURVEYS (OPCS) (1978), Occupational mortality 1970-1972, Londres, HSMO.

PAPPAS, G., S. QUEEN, W. HADDEN y G. FISHER (1993)," The increasing disparity in mortality between socioeconomic groups in the United States, 1960 and 1986", The New England Journal of Medicine, $\mathrm{n}^{\circ}$ 329, pp. 103-109.

REGIDOR, E., J.L. GUTIÉRREZ-FISAC y C. RODRÍGUEZ (1994), Diferencias y desigualdades en salud en España, Madrid, Ed. Díaz de Santos.

REGIDOR, E., S. DE MATEO, J.L. GUTIÉRREZ-FISAC y C. RODRÍGUEZ (1996), "Diferencias socioeconómicas en mortalidad en ocho provincias españolas", Medicina Clínica, n 106, 285-289.

REGIDOR E., J.L. GUTIÉRREZ-FISAC y C. RODRÍGUEZ (1995), "Increased socioeconomic differences in mortality in eight Spanish provinces", Social Science and Medicine, $\mathrm{n}^{\circ}$ 41, pp. 801-807.

REYNOLDS J.R. y C.E. ROSS (1998), "Social Stratification and Health: Education's Benefit Beyond Economic Status and Social Origins", Social Problems, n 45, pp. 221-247.

RODRÍGUEZ, J. A., y J. M. DE MIGUEL (1990), Salud y Poder, Madrid, CIS-Siglo XXI.

RODRÍGUEZ, J.A. y L. LEMKOW (1990), "Health and Social inequities in Spain", Social Science and Medicine, $\mathrm{n}^{\circ}$ 31, pp. 351-358.

ROSS, C.E. y CHIA-LING WU (1995), "The links between education and health", American Sociological Review, $\mathrm{n}^{\circ}$ 60, pp. 719-745.

SASCO, A.J., D. GRIZEAU, D. POBEL, O. CHATARD y M. DANZON (1994), "Tabaquisme et classe social en France de 1974 à 199l”, Bulletin Cancer, n 81, pp. 355-359.

SCHNALL, P., J. SCHWARTZ, P. LANDSBERGIS, K. WARREN y T. PICKERING (1992), "Relation between job strain, alcohol and ambulatory blood pressure", Hypertension, $\mathrm{n}^{\circ}$ 19, pp. 488-494. 
TOWSEND, P. y N. DAVIDSON (1982), Inequalities in Health. The Black Report, Penguin Books, Harmondsworth.

VAGERÖ, D. y O. LUNDBERG (1989), "Health inequalities in Britain and Sweden", Lancet, $\mathrm{n}^{\circ} 2$, pp. 35-36.

VAGERÖ, D. (1991), "Inequality in health. Some theoretical and empirical problems", Social Science and Medicine, $\mathrm{n}^{\circ} 32$, pp. 367-371.

VALKONEN, T. (1982), "Psychosocial stress and sociodemographic differentials in mortality from ischaemic heart disease in Finland", Acta Medica Scandinavia, n 660 (suppl), pp. 152-164.

(1989), "Adult mortality and level of education: A comparison of six countries", en J. Fox (Ed.), Health Inequalities in European Countries, pp.142-162. Gower, Aldershot.

(1993), "Problems in the measurement and international comparisons of socio-economic differences in mortality", Social Science and Medicine, $\mathrm{n}^{\circ} 36$, pp. 409-418.

VALKONEN T., T. MARTELEN y A. RIMPELÄ (1991), Socioeconomic mortality differences in Finland 1971-85, Helsinki, Central Statistical Office of Finland.

VAN REEK, J. y H. ADRIAANSE (1988), "Cigarette smoking cessation rates by level education in five western countries " (letter), International Journal of Epidemiology, $\mathrm{n}^{\circ}$ 17 , pp. 474-475.

WEST, P. (1991), "Rethinking the health selection explanation for health inequalities", Social Science and Medicine, $\mathrm{n}^{\circ}$ 32, pp. 373-378.

WHITEHEAD, M. (1988), "The Health Divide", en P. Townsend, N. Davidson y M. Whitehead (Ed.), Inequalities in Health. The Black Report and the Health Divide, pp. 215-381, Penguin Books, Harmondsworth.

\begin{abstract}
The objective of this study was to compare social inequalities in mortality by main causes of death in Spain and France during the 1988-90 period. In both Spain and France mortality in the manual workers category was always higher than that of the rest of the occupational groups for all causes. Farmers presented an intermediate level of mortality between professionals and managers and manual workers. The mortality of manual workers was 1.89 times higher than that of professionals and managers in Spain, and 1.67 higher in France. In Spain the greatest difference between the mortality of manual workers and professionals and managers was observed in accidents and respiratory diseases; in France, the greatest differences were observed in digestive diseases and respiratory diseases.
\end{abstract}

E-mail: llostao@unavarra.es 